\title{
Erratum to: Linkage and sequence analysis indicate that $C C B E 1$ is mutated in recessively inherited generalised lymphatic dysplasia
}

\author{
Fiona Connell · Kamini Kalidas • Pia Ostergaard - Glen Brice • \\ Tessa Homfray • Lesley Roberts • David J. Bunyan • Sally Mitton • \\ Sahar Mansour • Peter Mortimer - Steve Jeffery • Lymphoedema Consortium
}

Published online: 5 December 2009

(C) Springer-Verlag 2009

\section{Erratum to: Hum Genet}

DOI 10.1007/s00439-009-0766-y

Unfortunately, the wrong Fig. 6 was published in the online published article. Please see the correct figure 6 and the legend below.

The online version of the original article can be found under doi:10.1007/s00439-009-0766-y.

F. Connell · K. Kalidas · P. Ostergaard · S. Jeffery $(\bowtie)$

Medical Genetics Unit, Clinical Developmental Sciences,

St George's University of London, Cranmer Terrace,

London SW17 ORE, UK

e-mail:sggt100@sgul.ac.uk

G. Brice $\cdot$ T. Homfray $\cdot$ S. Mansour

South West Thames Regional Genetics Unit,

St George's University of London, Cranmer Terrace,

London SW17 ORE, UK

L. Roberts

Harris Birthright Unit, Obstetrics and Gynaecology,

King's College Hospital, Denmark Hill,

London SE5 9RS, UK

D. J. Bunyan

Wessex Regional Genetics Laboratory,

Salisbury District Hospital, Salisbury,

Wiltshire SP2 8BJ, UK

S. Mitton

Child Health, Clinical Developmental Sciences,

St George's University of London, Cranmer Terrace,

London SW17 0RE, UK

P. Mortimer

Cardiac and Vascular Sciences (Dermatology),

St George's University of London, Cranmer Terrace,

London SW17 ORE, UK

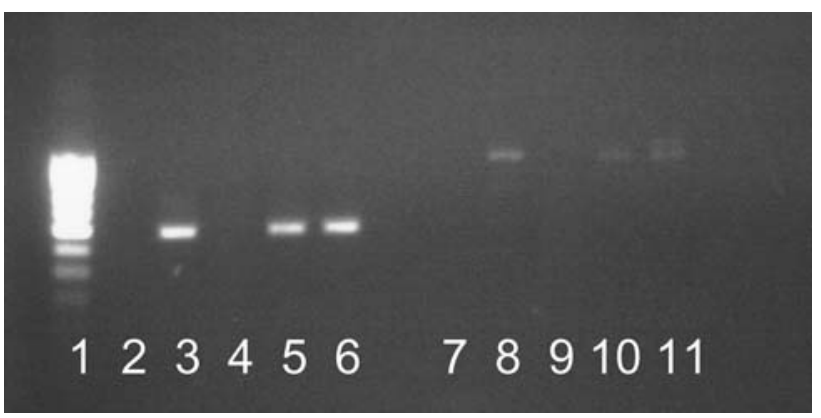

Fig. 6 cDNA from mother (I.1) amplified with two sets of primers for CCBE1 and run on an agarose gel. Lane 1 is a 100-kb ladder. Lanes 2 and 7 are negative controls. Lanes 3-6 are amplicons from primers in exons 1 and 6 (480 bp product). 3 and 6 are from skin biopsy of I.1, 4 is from lymphocytes of I.1, 5 is a control skin biopsy. Lanes 8-11 are amplified with primers from exons 1 and 11 (960 bp product). Lanes 8 and 11 are from skin biopsy of I.1, Lane 9 is from lymphocytes of I.1, Lane 10 is a control skin biopsy 